\title{
Progress of AFM single-cell and single-molecule morphology imaging
}

\author{
LI Mi $^{1,2}$, LIU LianQing $^{1 *}$, XI Ning ${ }^{1,3^{*}}$, WANG YueChao ${ }^{1}$, DONG ZaiLi $^{1}$, XIAO XiuBin $^{4}$ \& \\ ZHANG WeiJing ${ }^{4 *}$
}

\author{
${ }^{1}$ State Key Laboratory of Robotics, Shenyang Institute of Automation, Chinese Academy of Sciences, Shenyang 110016, China; \\ ${ }^{2}$ University of Chinese Academy of Sciences, Beijing 100049, China; \\ ${ }^{3}$ Department of Mechanical and Biomedical Engineering, City University of Hong Kong, Hong Kong, China; \\ ${ }^{4}$ Department of Lymphoma, Affiliated Hospital of Military Medical Academy of Sciences, Beijing 100071, China
}

\begin{abstract}
Atomic force microscopy (AFM) can probe single living cells and single native membrane proteins in natural fluid environments with label-free high spatial resolution. It has thus become an important tool for cellular and molecular biology that significantly complements traditional biochemical and biophysical techniques such as optical and electron microscopy and X-ray crystallography. Imaging surface topography is the primary application of AFM in the life sciences. Since the early 1990s, researchers have used AFM to investigate morphological features of living cells and native membrane proteins with impressive results. Steady improvements in AFM techniques for imaging soft biological samples have greatly expanded its applications. Based on the authors' own research in AFM imaging of living cell morphologies, a review of sample preparation procedures for single-cell and single-molecule imaging experiments is presented, along with a summary of recent progress in AFM imaging of living cells and native membrane proteins. Finally, the challenges of AFM high-resolution imaging at the single-cell and single-molecule levels are discussed.
\end{abstract}

atomic force microscopy, single-cell single-molecule, membrane protein, morphology, high-resolution imaging

Citation: $\quad$ Li M, Liu L Q, Xi N, et al. Progress of AFM single-cell and single-molecule morphology imaging. Chin Sci Bull, 2013, 58: 3177-3182, doi: $10.1007 / \mathrm{s} 11434-013-5906-\mathrm{z}$

Since its advent, atomic force microscopy (AFM) [1] has provided a means for investigating the physiological morphologies of single biomolecules and live single cells. The main drawback of conventional optical microscopy is a $>200 \mathrm{~nm}$ spatial resolution limit due to diffraction that prevents us from observing cellular ultra-structures. Although fluorescence microscopy can track the motion of single molecules, the results reflect only the labeled parts of the molecule [2] and those micro-environments that have direct impact on the biological function of the molecule [3] are not being observed. Electron microscopy requires that samples be fixed and dried, thus imaging living cells is not possible. An exception is environmental scanning electron microscopy (ESEM), but, at present, the image resolution is low

*Corresponding authors (email: lqliu@ sia.cn; xin@egr.msu.edu; zhangwj3072@163.com) and is still unable to image living cells [4]. X-ray crystallography can only provide static three-dimensional structures of proteins and cannot observe proteins dynamics [5]; a truly functional protein is dynamic by nature [6]. AFM has nanometer spatial resolution, it is easy to control, it can serially observe in fluids the dynamics of living cells and native membrane proteins (including the micro-environments around the membrane protein), and sample preparation is relatively simple. Because of these advantages, scholars worldwide have used AFM for research at the single-cell and single-molecule levels and have obtained unprecedented insights into biological processes that have led to new ideas and methods for disease diagnosis and drug discovery. After nearly 30 years of development, AFM has become a multifunctional toolbox [7]. In addition to imaging surface topographies with nanometer-scale resolution, it can also be 
used to characterize cellular mechanics, receptor-ligand binding forces, ligand distribution densities on cell surfaces, as well as the structure and mechanics of membrane proteins. Thus it importantly complements many other biochemical and biophysical techniques. Here, based on the authors' own research on AFM imaging of live cell, recent progress in AFM single-cell and single-molecule imaging is summarized and the current challenges of high-resolution imaging are discussed.

\section{Sample preparation techniques}

AFM uses a cantilever with a sharp tip at its end to raster scan the surface of a sample to construct a morphology image of the sample. The quality of AFM imaging is often related to the sample preparation, tip situation (tip radius, tip contamination), and the operator's experience. Of these three factors, sample preparation is often crucial. To obtain high-quality AFM images, one should avoid displacement of the sample caused by lateral forces exerted by the scanning probe; therefore, appropriate and nondestructive methods are needed to attach the sample to a solid substrate [8]. Methods for attaching cells to a substrate depend on the cell type. For adherent cells, this is very straightforward since they can be directly cultured on substrates such as Petri dishes and glass coverslips. After 1-2 d of culture, they tightly adhere to the substrate and can be imaged by AFM [9]. Poly- $L$-lysine is often coated on glass substrates to enhance adhesion [10].

Microbial cells such as bacteria and yeast do not naturally adhere to substrates and methods are needed to attach them. Two common techniques involve electrostatic adsorption and porous polymer membranes [11]. In electrostatic adsorption, positively charged macromolecules such as poly$L$-lysine are coated on the substrate so that the negatively charged microbial cells readily adsorb. Alternatively, cell suspensions filtered through a porous polymer membrane can trap cells in the pores; the polymer membrane is then attached to a solid substrate with double-sided adhesive tape. It should be noted that the porous polymer membrane immobilization method is based on commercial membranes and is thus best suited for round-shaped microbial cells whose sizes are comparable to the pore size. It is not well suited for rod-shaped cells.

These two immobilization methods (electrostatic adsorption and porous polymer membranes) are not applicable for suspended mammalian cells that are much larger than microbial cells and are relatively soft (microbial cells have firm cell walls). Researchers have used MEMS (microelectro-mechanical systems)-fabricated wells [12] to trap suspended mammalian cells. Although the wells can help cells to withstand the lateral forces of the scanning probe, there is nothing attaching the cells to the bottom of the well (i.e., no vertical immobilization). We developed an immobi- lization method combining the mechanical trapping by MEMS pillars with electrostatic adsorption with poly- $L$ lysine [13,14], thus immobilizing cells both horizontally and vertically. With this method, we have successfully imaged ultra-structures of living lymphoma cells.

AFM has been used to image the dynamics of the twodimensional structures of native membrane proteins [15], thus filling, to a certain extent, the gap between the static three-dimensional structure of a protein obtained by X-ray crystallography and its biological functions. While crystallography uses crystallized proteins purified by detergent, AFM directly investigates lipid bilayers containing reconstituted native proteins [16]. The lipid bilayer should be attached to substrates such as mica or highly ordered pyrolytic graphite that are atomically flat and very clean after stripping off the top layers [17]. Most of the time, physisorption is sufficient to attach the biological membrane onto the support and chemically modifying that are potentially hazardous to the molecules is not needed [18]. The interaction forces between the AFM tip and the protein membrane include long-range interaction forces (repulsive electrostatic double-layer forces) and short-range forces (attractive van der Waals forces). By adjusting the ionic strength and the $\mathrm{pH}$ of the buffer solution, the stronger electrostatic forces can be eliminated and therefore the membrane is tightly attached onto the substrate through van der Waals force [17].

Some bacteria have only a single type of protein in the outer membrane, such as bacteriorhodopsin in the "purple membrane" of Halobacterium salinarum [17]. The morphology of individual bacteriorhodopsin proteins can be imaged by AFM by extracting patches of purple membrane from the bacteria and attaching them to a substrate. The majority of cells have many different membrane proteins, therefore protein expression techniques are needed to create a cell membrane that has only one type of protein. The main expression systems are bacterial, yeast, insect cells, and mammalian cells [6]. After the target proteins are expressed in the cell membrane, patches of the membranes are suspended and then attached onto a substrate for AFM imaging [19]. Alternatively, the proteins can be purified from the expression cells and reconstituted in lipid membranes that are then attached to a substrate [15].

\section{$2 \quad$ AFM living cell imaging}

AFM imaging of living cells began in the early 1990s. Radmacher et al. [20] attached living human platelets onto a glass slide and imaged different stages of their activation. Henderson et al. [21] cultured glial cells on glass coverslips that were coated with collagen and serially observed the dynamics of actin filaments with time-lapse AFM, demonstrating that the dynamics of cellular ultra-structures can be investigated. After 20 years of development, AFM cell im- 
aging achieved great progress. AFM imaging mode for cell imaging has developed from contact mode to several other modes (tapping mode [22], Magnetic AC mode [23], peak force tapping mode [24]); High-speed AFM techniques are improving [2,5]; Living mammalian suspended cells can be imaged by AFM $[13,14]$. The research hot of AFM cell imaging in recent years focused on combining fluorescence microscopy with AFM to investigate the cellular ultra-structures and using time-lapse AFM to serially observe the dynamics of the surface ultra-structures during cellular physiological activities. Fantner et al. [25] used high-speed AFM to investigate the dynamics of cellular surface structures after stimulation by the antimicrobial peptide CM15, and also established the relationship between cell death and cellular morphological changes with combined AFM and fluorescence microscopy. Kirmse et al. [26] used combined AFM and fluorescence microscopy to investigate cell adhesion, force generation and extracellular proteolysis in cell metastasis by culturing melanoma cells (expressed MT1MMP protease) on fibrillar collagen-I matrix-coated mica (the matrix was FITC-labeled). The results indicated that cells can pull collagen fibers into bundles and cleave them; the cleaving process was impeded by either blocking the integrin or adding cytochalasin D and blebbistatin, thus revealing the interdependency of cell adhesion, force generation and extracellular proteolysis during cell metastasis.
El-Kirt-Chatel et al. [27] combined fluorescence and AFM to investigate the morphological changes of macrophages (ruffles, lamellipodia, filopodia, membrane remnants, phagocytic cups) infected by candida. This was performed for the different stages of infection where candida changed from yeast to hyphae that escaped from the phagocytes, thus providing new insights into the candida-macrophage interaction with respect to changes in cell ultrastructure.

As mentioned above, we have developed a cell immobilization method based on mechanical trapping by silicon pillars and electrostatic adsorption by poly-L-lysine $[13,14]$. As shown in Figure 1, the surface morphology of living lymphoma (Raji) cells was imaged by AFM by this method. The silicon pillars were fabricated with MEMS technology, and poly- $L$-lysine was coated on the chip. After dropping the Raji cell suspension onto the chip, the chip was attached to a glass slide with double-sided adhesive tape and placed in a Petri dish containing phosphate-buffered saline (PBS). The pillars help the cell to withstand lateral forces exerted by the scanning probe in the horizontal direction and poly$L$-lysine attaches the cell to the bottom of the chip. The spring constant of the cantilever was $0.12 \mathrm{~N} \mathrm{~m}^{-1}$. The imaging was performed in tapping mode at a drive frequency of $9 \mathrm{kHz}$. The scan rate was $0.3 \mathrm{~Hz}$. Figure 1 (a) illustrates cell immobilization by the four pillars. Figure 1 (b) is a photo of the pillar chip, Figure 1(c) is a SEM image of the
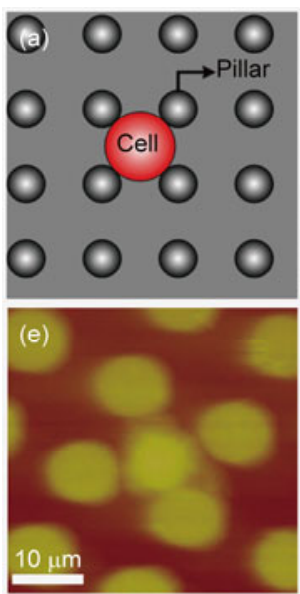

(j)

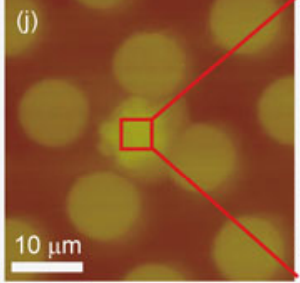

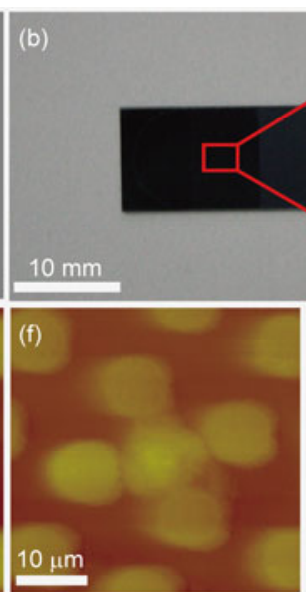

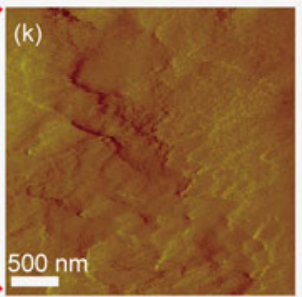

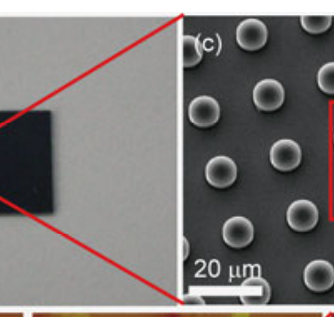
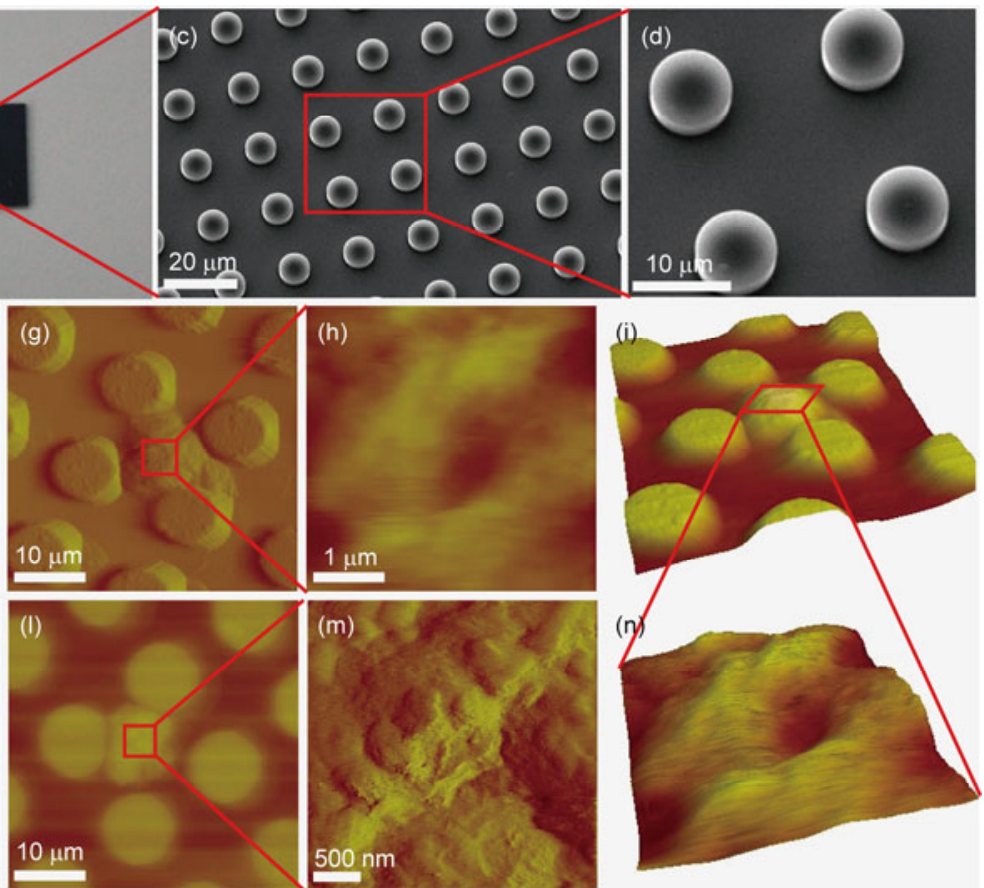

Figure 1 AFM imaging of lymphoma Raji cells immobilized by pillars. (a) Schematic of immobilizing cells by pillars; (b) optical image of the MEMS-fabricated pillar chip; (c) SEM image of the pillar array; (d) SEM image of the pillar unit; (e-i, n) tapping-mode AFM images of live lymphoma Raji cells in PBSn. (e) Height image; (f) phase image and (g) amplitude image of the whole cell; (h) height image of the local cellular area; (i) three-dimensional image of the whole cell; (n) three-dimensional image of the local cellular area. Reprinted (with some modification) with permission from [13]. Copyright 2011 Elsevier Inc. (j, k) Cellular morphology after 2-h stimulation by $0.2 \mathrm{mg} \mathrm{mL} \mathrm{m}^{-1}$ Rituximab. (j) Height image of the whole cell; (k) height image of the local cellular area. (1, m) Cellular morphology after 2-h stimulation by $0.5 \mathrm{mg} \mathrm{mL} \mathrm{mL}^{-1}$ Rituximab. (l) Height image of the whole cell; (m) height image of the local cellular area. Reprinted (with some modification) with permission from [14]. Copyright 2012, Editorial office of Acta Physico-Chimica Sinica. 
pillar array, and Figure 1 (d) is an SEM image of a fourpillar unit. The pillar height was $5 \mu \mathrm{m}$, and the pillar diameter was $10 \mu \mathrm{m}$. Because of variable cells sizes, the distance between the pillars was designed to accommodate three sizes $(5,10,15 \mu \mathrm{m})$. Figure 1 (e)-(i) are AFM height, phase, amplitude, and three-dimensional images of a living lymphoma (Raji) cell, respectively. The scan range was $40 \mu \mathrm{m}$. Figure $1(\mathrm{~h})$ and (n) are the height and three-dimensional images of the cell surface, respectively (scan range $=4 \mu \mathrm{m}$ ). From the high-quality AFM images, one can see that the live cell was tightly immobilized. Based on the pillar's immobilization, we were able to observe changes in the surface ultra-structures of the Raji cells after stimulation by different concentrations of Rituximab, a monoclonal antibody-targeted drug that can bind to CD20 on B cells and cause apoptosis. From the AFM images (Figure 1(k) and $(\mathrm{m})$ ), we can see that the cell surface became corrugated and rough after Rituximab stimulation. In addition, as the Rituximab concentration increased, the cell topography changed more significantly and some tubercles appeared. These images improve our understanding of Rituximab's anti-cancer effect.

\section{AFM membrane protein imaging}

Membrane proteins account for about $30 \%$ of human proteins, they play a crucial role in cell physiological activities, and about half of all approved drugs target membrane proteins [28]. However, because of the challenges in the production, purification, and crystallization of membrane proteins, their structures are poorly understood [29]. Of the more than 65000 structures in the RCSB Protein Data Bank, fewer than a dozen are full-size human membrane proteins [28]. Although there have been many new advances on resolving protein structures $[30,31]$, there are still many difficulties waiting to be addressed. With AFM, one can directly image two-dimensional structures of fully functional, native membrane proteins reconstituted in lipid bilayers and therefore obtain complementary structural information [16]. Imaging membrane proteins began in 1995 when Muller et al. [32] attached purple membranes onto mica and imaged the morphology of the single bacteriorhodopsin molecules in an

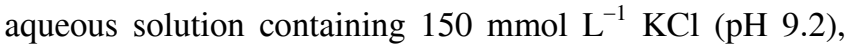
revealing bacteriorhodopsin trimers. In vertebrate retinal photoreceptors, the rod disc membranes contained densely packed rhodopsin molecules and to investigate how these molecules were organized in the disc membrane, Fotiadis et al. [33] attached the mouse disc membranes to mica and imaged the morphology of the rhodopsin molecules in the disc membranes, revealing that rhodopsin molecules existed in dimers.

The research hot of AFM membrane protein imaging in recent years was observing the dynamics of the membrane protein's two-dimensional structures. Mari et al. [15] observed conformation changes of MlotiK1 potassium chan- nels that were expressed and purified from E. coli cells and then reconstituted into lipid membranes. As shown in Figure 2(a)-(c), they revealed that the MlotiK1 potassium channel had a fourfold symmetric arrangement forming a pore-like vestibule after binding of cAMP; the channel closed the vestibule in the absence of cAMP binding and reopened it after re-binding cAMP. Shibata et al. [34] observed light-induced conformation changes of bacteriorhodopsin molecules, showing that the bacteriorhodopsin existed in trimers without illumination, while after illumination each monomer was brought into contact with adjacent trimers (Figure 2(d)-(g)). Casuso et al. [35] first observed conformation changes of ATP-ase c-rings in the purple membrane (dimer-monomer-dimer), revealing membranemediated protein-protein interactions of bacteriorhodopsin with the ATP-ase (Figure 2(h)-(n)). These studies directly showed conformational changes of single membrane proteins and provided new insights concerning the structure and function of membrane proteins that were complementary to the static three-dimensional structures from X-ray crystallography. With high-speed AFM, one can observe molecular activities with nanometer resolution at sub-100ms timescales, thus providing biologists with unprecedented visualization of functional proteins without having to model the dynamics [2]. It can be anticipated that high-speed AFM will have a significant impact in the life sciences.

\section{Challenge and outlook}

AFM is an important complement to traditional biochemical and biophysical techniques. Because of increasingly wider applications of AFM in life sciences, we now have a deeper understanding of physiological activities at the single-cell and single-molecule levels [36-40]. We can now obtain highresolution images of single membrane proteins and observe conformational changes. However, it should be noted that these lipid bilayers are not a realistic micro-environment of the protein since many different proteins and lipids coexist in a cell membrane. The difference of micro-environments may influence the structure and function of proteins and therefore the imaging results may not completely reflect the behaviors of the membrane protein in living cells. Currently, the spatial resolution for AFM imaging of whole mammalian cells is only about $50 \mathrm{~nm}$ [41]; this is because the cell membranes are soft, dynamic, and corrugated in nature, which in turn causes the scanning tip to deform the cell membrane. In addition, there are adhesive interactions between the AFM tip and the cell membrane. All of these factors seriously degrade the resolution of AFM imaging of whole mammalian cells and so far we have not been able to resolve single proteins on living cells. In the case of hard materials, atomic spatial resolution with AFM has been demonstrated [42], but to obtain comparable images of living cells, the complex, soft materials and the liquid environments 

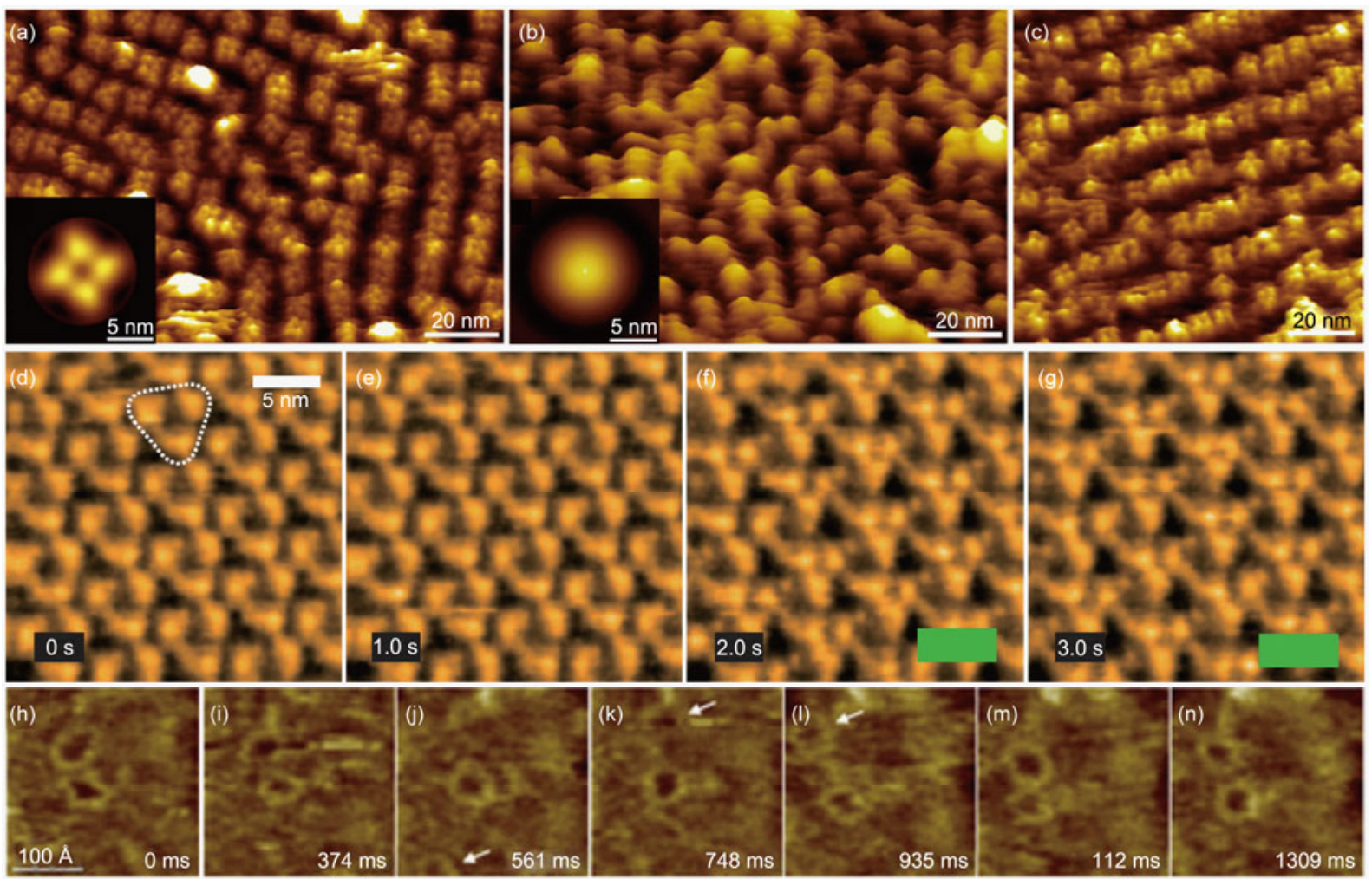

Figure 2 AFM imaging of membrane proteins. (a-c) cAMP-induced conformational changes of MlotiK1 potassium channel. Reprinted with permission from [15]. Copyright 2011 National Academy of Sciences. (a) After the binding of cAMP, the MlotiK1 potassium channel is a fourfold symmetric pore-like structure (open). (b) Without cAMP, the MlotiK1 potassium channel is closed. (c) After re-adding cAMP, the MlotiK1 potassium channel reopened. (d-g) Illumination-induced conformation changes of bacteriorhodopsin. Reprinted with permission from [34]. Copyright 2010 Macmillan Publishers Ltd. (d, e) Without illumination, bacteriorhodopsin forms trimers. (f, g) With illumination, each monomer moves into contact with adjacent trimers. (h-n) Conformational changes of the ATP-ase c-rings in the purple membrane. The conformation changed (dimer(h)-monomer(k)-dimer(n)). Reprinted with permission from [35]. Copyright 2010 Biophysical Society.

present significant challenges that must be overcome. Researchers have presented many cell immobilization methods, but the methods that can effectively inhibit the movement of cell membrane are urgently needed. How to improve the imaging resolution in complex environments has become a major challenge that hinders the further development of AFM imaging. It takes several minutes to obtain one image with conventional AFM, which is much longer than the timescale of many cell processes. Although high-speed AFM can be used to observe the dynamics of single molecules, it usually involves small, flat samples. To apply high-speed imaging to living cells (large, curved surfaces), many technical and conceptual obstacles must be overcome [43]. The label-free advantage of AFM becomes a weakness in complex systems because many proteins are indistinguishable from one another in an AFM image [2]. One solution to this problem is to attach biological molecules onto the AFM tip for specific molecular recognition. However, tip functionalization is complex and time-consuming, accurate data collection and interpretation remain technically challenging, it is difficult to standardize the experimental process, and it is difficult to avoid tip alterations or contamination during scanning $[44,45]$.

We can fully explore the potential of AFM if we combine AFM with other techniques that simultaneously obtain complementary information on cellular physiological activities. By combining AFM with fluorescence microscopy [26,27], we integrate the high spatial resolution of AFM with specific fluorescence labeling that allows identification of specific proteins and cellular processes. By combining AFM with a patch clamp [46], we can simultaneously obtain cell membrane movement and ion channel current. AFM is basically a surface tool and can not detect the internal structures of the cells. Based on scanning probe microscopy (SPM) technique, researchers have presented scanning near-field ultrasonic holography (SNFUH) [47] which is capable to detect the buried structures and successfully imaged the malaria parasites in red blood cells. In summary, dramatic progress has made AFM a valuable tool in the life sciences since its introduction nearly 30 years ago. But AFM still have many difficulties and challenges that require researchers from different disciplines to work together to solve and therefore deepen and widen the AFM researches in life sciences. 
This work was supported by the National Natural Science Foundation of China (61175103) and CAS FEA International Partnership Program for Creative Research Teams.

1 Binnig G, Quate C F, Gerber C. Atomic force microscope. Phys Rev Lett, 1986, 56: 930-933

2 Katan A J, Dekker C. High-speed AFM reveals the dynamics of single biomolecules at the nanometer scale. Cell, 2011, 147: 979-982

3 Hinterdorfer P, Garcia-Parajo M F, Dufrene Y F. Single-molecule imaging of cell surfaces using near-field nanoscopy. Acc Chem Res, 2012, 45: 327-336

4 Muscariello L, Rosso F, Marino G, et al. A critical overview of ESEM applications in the biological field. J Cell Physiol, 2005, 205: 328-334

5 Kodera N, Yamamoto D, Ishikawa R, et al. Video imaging of walking myosin $\mathrm{V}$ by high-speed atomic force microscopy. Nature, 2010, 468: 72-76

6 Robertson J W F, Kasianowicz J J, Banerjee S. Analytical approaches for studying transporters, channels and porins. Chem Rev, 2012, 112: 6227-6249

7 Muller D J, Dufrene Y F. Atomic force microscopy as a multifunctional molecular toolbox in nanobiotechnology. Nat Nanotechnol, 2008, 3: 261-269

8 Kirat K E, Burton I, Dupres V, et al. Sample preparation procedures for biological atomic force microscopy. J Microsc, 2005, 218: 199-207

9 Matzke R, Jacobson K, Radmacher M. Direct, high-resolution measurement of furrow stiffening during division of adherent cells. Nat Cell Biol, 2001, 3: 607-610

10 Puntheeranurak T, Wildling L, Gruber $\mathrm{H} \mathrm{J}$, et al. Ligands on the string: Single-molecule AFM studies on the interaction of antibodies and substrates with the $\mathrm{Na}^{+}$-glucose co-transporter SGLT1 in living cells. J Cell Sci, 2006, 119: 2960-2967

11 Dufrene Y F. Atomic force microscopy and chemical force microscopy of microbial cells. Nat Protoc, 2008, 3: 1132-1138

12 Rosenbluth M J, Lam W A, Fletcher D A. Force microscopy of nonadherent cells: A comparison of leukemia cell deformability. Biophys J, 2006, 90: 2994-3003

13 Li M, Liu L, Xi N, et al. Imaging and measuring the rituximab-induced changes of mechanical properties in B-lymphoma cells using atomic force microscopy. Biochem Biophys Res Commun, 2011, 404: 689-694

14 Li M, Liu L, Xi N, et al. Drug-induced changes of topography and elasticity in living B lymphoma cells based on atomic force microscopy. Acta Phys Chim Sin, 2012, 28: 1502-1508

15 Mari S A, Pessoa J, Altieri S, et al. Gating of the MlotiK1 potassium channel involves large rearrangements of the cyclic nucleotide-binding domains. Proc Natl Acad Sci USA, 2011, 108: 20802-20807

16 Fotiadis D. Atomic force microscopy for the study of membrane proteins. Curr Opin Biotechnol, 2012, 23: 510-515

17 Muller D J, Engel A. Atomic force microscopy and spectroscopy of native membrane proteins. Nat Protoc, 2007, 2: 2191-2197

18 Muller D J. Adsorption of biological molecules to a solid support for scanning probe microscopy. J Struct Biol, 1997, 119: 172-188

19 Muller D J, Hand G M, Engel A, et al. Conformational changes in surface structures of isolated connexin 26 gap junctions. EMBO J, 2002, 14: 3598-3607

20 Radmacher M, Tillmann R W, Fritz M, et al. From molecules to cells: Imaging soft samples with the atomic force microscope. Science, 1992, 257: 1900-1905

21 Henderson E, Haydon P G, Sakaguchi D S. Actin filament dynamics in living glial cells imaged by atomic force microscopy. Science, 1992, 257: 1944-1946

22 Vie V, Giocondi M C, Lesniewska E, et al. Tapping-mode atomic force microscopy on intact cells: Optimal adjustment of tapping conditions by using the deflection signal. Ultramicroscopy, 2000, 82: 279-288

23 Chen P P, Dong H T, Chen L, et al. Application of atomic force mi- croscopy to living samples from cells to fresh tissues. Chin Sci Bull, 2009, 54: 2410-2415

24 Alsteens D, Dupres V, Yunus S, et al. High-resolution imaging of chemical and biological sites on living cells using peak force tapping atomic force microscopy. Langmuir, 2012, 28: 16738-16744

25 Fantner G E, Barbero R J, Gray D S, et al. Kinetics of antimicrobial peptide activity measured on individual bacterial cells using highspeed atomic force microscopy. Nat Nanotechnol, 2010, 5: 280-285

26 Kirmse R, Otto H, Ludwig T. Interdependency of cell adhesion, force generation and extracellular proteolysis in matrix remodeling. J Cell Sci, 2011, 124: 1857-1866

27 El-Kirt-Chatel S, Dufrene Y F. Nanoscale imaging of the candidamacrophage interaction using correlated fluorescence-atomic force microscopy. ACS Nano, 2012, 6: 10792-10799

28 Baker M. Making membrane proteins for structures a trillion tiny tweaks. Nat Methods, 2010, 7: 429-433

29 Bill R M, Henderson P J F, Iwata S, et al. Overcoming barriers to membrane protein structure determination. Nat Biotechnol, 2011, 29: 335-340

30 Zhang X, Ren W, Decaen P, et al. Crystal structure of an orthologue of the NaChBac voltage-gated sodium channel. Nature, 2012, 486: 130-134

31 Ma D, Lu P, Yan C, et al. Structure and mechanism of a glutamateGABA antiporter. Nature, 2012, 483: 632-636

32 Muller D J, Schabert F A, Buldt G, et al. Imaging purple membranes in aqueous solutions at sub-nanometer resolution by atomic force microscopy. Biophys J, 1995, 68: 1681-1686

33 Fotiadis D, Liang Y, Filipek S, et al. Rhodopsin dimers in native disc membranes. Nature, 2003, 421: 127-128

34 Shibata M, Yamashita H, Uchihashi T, et al. High-speed atomic force microscopy shows dynamic molecular processes in photoactivated bacteriorhodopsin. Nat Nanotechnol, 2010, 5: 208-212

35 Casuso I, Sens P, Rico F, et al. Experimental evidence for membranemediated protein-protein interaction. Biophys J, 2010, 99: L47-L49

36 Li M, Liu L Q, Xi N, et al. Atomic force microscopy imaging and mechanical properties measurement of red blood cells and aggressive cancer cells. Sci China Life Sci, 2012, 55: 968-973

37 Li M, Liu L Q, Xi N, et al. Detecting CD20-Rituximab interaction forces using AFM single-molecule force spectroscopy. Chin Sci Bull, 2011, 56: 3829-3835

38 Li M, Liu L Q, Xi N, et al. Imaging and mechanical property measurement of the lymphoma cells by atomic force microscopy (in Chinese). Chin Sci Bull (Chin Ver), 2010, 55: 2188-2196

39 Li M, Xiao X, Liu L, et al. Imaging and measuring the molecular force of lymphoma pathological cells using atomic force microscopy. Scanning, 2013, 35: 40-46

40 Li M, Liu L Q, Xi N, et al. Mapping CD20 molecules on the lymphoma cell surface using atomic force microscopy. Chin Sci Bull, 2013, 58: 1516-1519

41 Muller D J, Dufrene Y F. Force nanoscopy of living cells. Curr Biol, 2011, 21: R212-R216

42 Gross L, Mohn F, Moll N, et al. The chemical structure of a molecule resolved by atomic force microscopy. Science, 2009, 325: 1110-1114

43 Casuso I, Rico F, Sheuring S. High-speed atomic force microscopy: Structure and dynamics of single proteins. Curr Opin Chem Biol, 2011, 15: 704-709

44 Heinisch J J, Lipke P N, Beaussart A, et al. Atomic force microscopylooking at mechanosensors on the cell surface. J Cell Sci, 2012, 125: 4189-4195

45 Dufrene Y F, Evans E, Engel A, et al. Five challenges to bringing single-molecule force spectroscopy into live cells. Nat Methods, 2011, 8: $123-127$

46 Zhang P C, Keleshian A M, Sachs F. Voltage-induced membrane movement. Nature, 2001, 413: 428-432

47 Shekhawat G S, Dravid V P. Nanoscale imaging of buried structures via scanning near-field ultrasound holography. Science, 2005, 310: 89-92

Open Access This article is distributed under the terms of the Creative Commons Attribution License which permits any use, distribution, and reproduction in any medium, provided the original author(s) and source are credited. 